\title{
Intention-Based Walking Support for Paraplegia Patients with Robot Suit HAL
}

\author{
Kenta Suzuki ${ }^{1}$, Gouji Mito ${ }^{1}$, Hiroaki Kawamoto ${ }^{2}$, \\ Yasuhisa Hasegawa ${ }^{1}$ and Yoshiyuki Sankai ${ }^{1}$ \\ ${ }^{1}$ Graduate School of Systems and Information Engineering, University of Tsukuba \\ ${ }^{2}$ Japan Association for the Advancement of Medical Equipment \\ Tennodai 1-1-1, Tsukuba, 305-8573, Japan \\ Email: cybernoid@golem.kz.tsukuba.ac.jp
}

\begin{abstract}
This paper proposes an algorithm to estimate human intentions related with walking in order to comfortably and safely support a paraplegia patient's walk. A robot suit "HAL" has been developed for an enhancement of healthy person's activities and for support of physically challenged person's daily life. Assisting method based on bioelectrical signals such as myoelectricity successfully supports healthy person's walking. These bioelectrical signals, however, cannot be measured properly from a paraplegia patient. Therefore another interface that can estimate patients' intentions without any manual controller are desired for robot control since a manual controller deprives a patient of his/her hands' freedom. Estimation of patients' intentions contributes to support not only comfortably but also safely, because an inconformity between the robot suit motion and the patient motion results in his/her stumbling or falling. The proposed algorithm, therefore, estimates patient's intentions from a floor reaction force reflecting patient's weight shift during walking and standing. The effectiveness of this algorithm is investigated through experiments on a paraplegia patient who has a sensory paralysis on both legs, especially his left leg. We show that HAL supports patient's walk properly, estimating his intentions based on floor reaction force.

Keywords: robot suit, paraplegia, walking support, intention estimation, floor reaction force
\end{abstract}

\section{INTRODUCTION}

People may have muscle rigidity, relaxation, involuntary contraction of muscle, and sensory paralysis due to cerebral paralysis, stroke, spinal cord injury, muscular dystrophy and postpolio syndrome. Even if people do not suffer from these physical problems, aging brings various troubles on his/her motility. Most people who have problems on the lower limbs due to these symptoms or aging are unable to walk and are bedridden all day long at worst. Moreover, this situation depresses the patients' feelings, for instance bedridden patients lose his/her life worth living. Caregivers including the patient's family also receive hard works to look after him/her, once a person has a trouble in the motility. To relieve these problems and to support the patient's independent life, it is quite important to provide a safe and convenient transportation device. A wheelchair is now used in most cases as a transportation device for 
patients with gait disorder. It is convenient for the patients because they can move easily as long as an enough muscular power is left in their upper body. Even if a patient has weakness of the arms, a motorized wheelchair could be used. However, wheelchairs have some problems in its using environment and the user's posture. Especially, wheelchair users are apt to keep sitting posture for a long time and have less opportunities to exercise their own lower bodies. That may cause a decrease in not only muscular power of lower body with paralysis but also residual physical functions. This problem could be solved if a patient with paraplegia could walk on his/her legs as a healthy person does. Therefore, a device which helps a patient walk in his/her standing posture would be one of the solutions since he/she can locomote with his/her leg receiving a physical support. Several devices for walking support have been developed. In our study, a wearable type robot "Robot suit HAL (Hybrid Assistive Limb)" has been developed in order to physically support wearer's daily activities and heavy works. HAL-1 utilizing DC motors and ball screws shown in Fig. 1(a) was developed as the first prototype of HAL [1], and it enhanced wearer's walking by amplifying wearer's own joint torque. After developing some prototypes, HAL-3 shown in Fig. 1(b) was developed toward a more suitable system to be used in actual daily life [2,3]. These robot suits have a power unit on each hip and knee joint, and they support functional motions of lower limbs with multiple joints simultaneously. After that, HAL-5 (see Fig. 1(c)), that is demonstrated at the 2005 World Exposition in Aichi, has been developed for whole body support. It assists human motions involving wearer's upper-body activities such as carrying heavy loads. Meanwhile, "RoboKnee" [4] and "Wearable Walking Helper" [5] have been developed to support the knee motion by using linear actuators. However, it is difficult for these two devices to support a patient with paraplegia since these devices cannot support their multiple joints in lower limbs simultaneously. As an exoskeleton to assist soldiers, disaster relief workers and other emergency personnel who needs to move long distance on foot on their fields, Kazerooni et al., $[6,7]$ has developed "BLEEX" that supports human's walking while carrying heavy loads on his/her back. This exoskeleton is not designed for welfare purposes, and it is too large and heavy (75 kg including exoskeleton weight and maximum payload) for patients to handle as their own supporting devices in actual daily life. To provide effective physical support according to each wearer's condition, it is necessary to strongly focus on control algorithm as well as mechanism of supporting devices. The robot suit HAL has a cybernic control system that is a hybrid control algorithm consisted of "Cybernic voluntary control (Bio-cybernic control)" and "Cybernic autonomous control (Cybernic robot control)". The cybernic control system can provide suitable physical support to wearers in various conditions such as a healthy person, a physically challenged person and so on by using two algorithms as complementary controls.

The features of each control algorithm are described below. The cybernic voluntary control provides physical support according to his/her voluntary muscle activity. Power units of HAL generate power assist torque by amplifying wearer's own joint torque estimated from his/her bioelectrical signals, and the support motions are consequently controlled by wear's signal adjustment. This control was used for power assist of healthy person's activities [8], for example walking and standing up from sitting posture, and we confirmed the cybernic voluntary control successfully supported a wearer's motion. The bioelectrical signals including myoelectricity are useful and reliable information to estimate human's motion intentions because the signals are measured just before corresponding muscle activities. Thus, the wearers receive the physical support directly by unconscious interface using the bioelectrical signals, which realize much more easily operation than manual controllers such as a joystick. HAL 


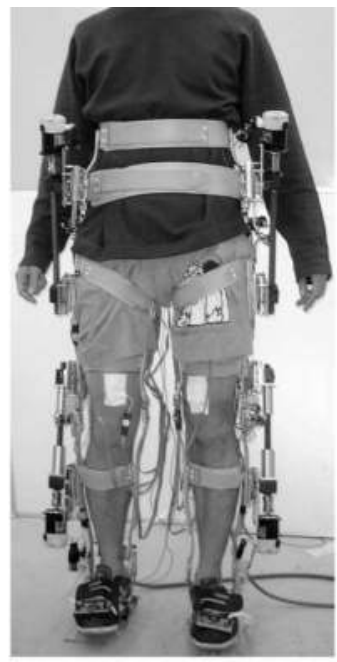

(a) HAL-1 (1999)

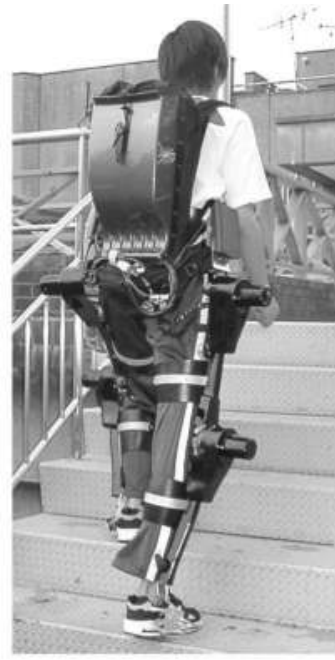

(b) HAL-3 (2001)

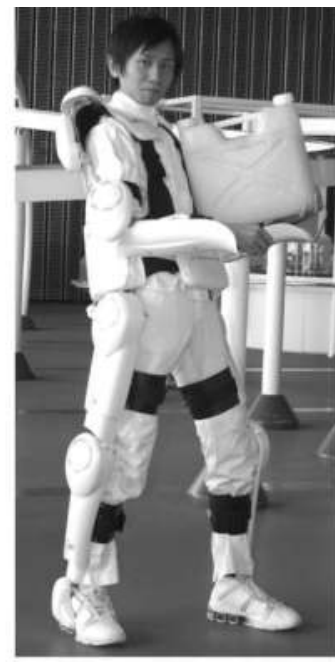

(c) HAL-5 (2005)

Fig. 1. Representative conventional robot suits we have developed. (a) and (b) HAL supports wearer's lower body motion. (c) HAL supports their whole body motion. A twenty kilogram load is carried on wearer's single arm.

can physically support patients with some handicaps on their lower limbs as well as healthy people because HAL supports functional motions with multiple joints simultaneously, covering whole of lower limbs. However, as a whole, a patient with gait disorder is not able to receive walking support by the cybernic voluntary control because the signals that induce a broken walking pattern are not used for the power assist, and no signal is observed in the severest case. In that case, the cybernic autonomous control can provide an effective physical support.

The cybernic autonomous control autonomously provides a desired functional motion generated according to wearer's body constitutions, conditions and purposes of motion support. While the bioelectrical signals are mainly used in the cybernic voluntary control, various kinds of information except for the bioelectrical signals, such as reaction force and joint angle can be used to provide comfortable physical supports. It can be applied to rehabilitation and walking support for the patients as well as power assist for healthy people and it enables HAL to be used as alternate body functions for their handicaps or weakness of muscular power. In that case, HAL needs to observe wearer's conditions and motion intentions from any motion information instead of his/her bioelectrical signals in order to provide a suitable support with a suitable moment. HAL-3 with the cybernic autonomous control successfully enhances healthy person's walking, stair-climbing, standing up from sitting posture and cycling, synchronizing with his/her body conditions [9]. In that work, floor reaction forces and joint angles are used as motion information to detect wearer's conditions. Posture control as well as sensing and recognition for environment including a wearer is essential technologies for an entirely autonomous physical support, but they remain to be solved. In this paper, the cybernic autonomous control among the cybernic control system is applied to the robot suit HAL in order to support a paraplegia patient's walk. Our conventional cybernic autonomous control 
algorithm [9] cannot be applied to them directly due to variety of patients' body constitutions and handicaps. Generally, the human intentions in his/her mind are essentially independent from the physical interactions between a body and an environment. As far as we know, no current technologies can directly measure and extract the human intentions. However, we can sometimes guess the human intentions in his/her mind from his/her appearances or motions. Besides, we can estimate his/her corresponding intentions if we observe a motion or an appearance that is closely connected with his/her intentions. According to conventional works on human transient walking [10,11], a COG shift to one leg is prior motion to a walk. That motion is an indispensable to swing a leg and can be observed earlier than a bioelectrical signal such as myoelectricity, because it is observed before a human starts swinging a leg, while a bioelectrical signal is observed when corresponding muscles start contracting. The COG shift can be used for an early and smart trigger to start walking supports, because the shift is involved into preliminary motions for a walk and human does not have to operate any manual switch to start the walking supports. On the other hand, gait stopping is similar to the time-reverse motion of the gait initiation, and the COG stops at around the center of both supporting legs. Therefore this paper proposes an intention estimator that can estimate his/her walking intentions from the COG shift that is closely connected with his/her intention. We define that intention-based support (including the walking support) is to provide a physical support for the next wearer's desired motion that can be predicted based on the current state or motion induced by his/her intention. In a case of walking, a human shifts the COG to a supporting leg side before he/she starts swinging a leg. If the robot suit HAL can sense the COG shift induced by his/her intention, it can predict his/her walking start and then start walking support. Our project aims to realize the comfortable walking supports for paraplegia patients that reflect the patients' intentions on the start and stop of walking, cycle and stride of walking motion, walking direction and so on. We call the walking support conforming to these various intentions of walking "Intention-based walking support". It is hoped that the intention-based walking support improves the usability, safety and reliability of the robot suit HAL. As the first step, this paper focuses on three kinds of intentions: start and stop of walking and the beginning to swing a leg, and proposes a control algorithm that uses patient's residual physical functions effectively. We need to observe not only the COG shift in a lateral plane but also the forward COG shift and bending of the upper body in order to distinguish the gait initiation from other similar motions such as just stepping or changing a supporting leg for a leg relaxation. However, the robot suit HAL can understand his/her intention if we instruct the wearer to shift the COG to either of his/her legs in order to receive the physical support for swinging a leg. Therefore, floor reaction force can be one of reliable information that reflects his/her intentions without any manual interfaces if a patient can control his/her weight balance in lateral plane by holding a walking frame with own hands. The purpose of this study is that HAL helps a patient with paraplegia walk in a standing posture. Based on our conventional works, two additional functions should be developed for this purpose. First, HAL should generate a suitable bipedal walk according to patient's body constitutions. Reference trajectories for each joint support should be designed in another way because the bioelectrical signals are not observed from a patient with paraplegia. The reference motions consist of swinging wearer's leg, supporting his/her weight and shifting his/her weight from one leg to the other. Second, HAL should provide walking support according to patient's intentions that are estimated from wearer's COG shift. To achieve two functions mentioned above, this paper takes the following approaches. They are: 
1. To achieve the bipedal locomotion partially based on walking patterns of a healthy person,

2. To estimate wearer's intentions from his/her COG shift that is observed by the floor reaction force and

3. To synchronize support motions with estimated wearer's intentions: the walk start, stop and the beginning to swing a leg.

The following section explains assumptions and approach of this study. Section 3 introduces the robot suit "HAL-5 Type-C" used in this experiment. Section 4 describes the proposed algorithm for walking support and intention estimation. Section 5 shows experimental results and verifies the performance of the proposed algorithm in HAL-5 Type-C. Finally, section 6 is the conclusion.

\section{ASSUMPTIONS AND APPROACH}

In this paper, a proposed algorithm is applied to the walking support for a paraplegia patient called "subject A" in this paper. He has sensory paralysis on both legs, especially left leg because of spinal cord injury by traffic accident. He can keep standing posture and slowly walk by himself with two canes. In this case, we cannot measure proper bioelectrical signals to estimate his intention during walking because of disorder of neural transmission. We, therefore, use floor reaction force instead of the bioelectrical signals in this experiment. Floor reaction force (FRF) reflects his weight shift during walking and standing. It should be noted that he can control his balance holding a walking frame and that our algorithm can estimate his intentions from his FRF. That is our algorithm synchronizes the physical support with his intentions through his controlled weight balance by using not any manual controllers such as a joystick but FRF during walking and standing. The reference patterns to the patient are extracted from healthy person's walk. The healthy person's walking motion could be suitable to the patient if he/she has the same body constitution as the healthy person. The extracted walking motion, however, should be adjusted according to the patient's body constitution and handicap conditions, for example a walking cycle and amplitude of each joint trajectory in swinging a leg.

\section{ROBOT SUIT HAL}

In the experiment, the robot suit HAL-5 clinical type (HAL-5 Type-C) which is made for the subject $\mathrm{A}$ is used. Figure 2 shows the overview of HAL-5 Type-C and Fig. 3 is its system configurations. As in the case of the conventional type of HAL (HAL-3), HAL-5 Type-C consists of power units, exoskeletal frames, sensors and a controller. Power units are attached on each hip and knee joints and actuate each joint by their torques. On ankle joints, springs are attached so that wearer's ankle joints could come back to a normal angle even if any external forces do not affect the joints. The spring action contributes to avoiding collisions between a toe of a swing leg and a floor. The exoskeletal frames are fixed to wearer's legs with molded plastic bands, and transmit torques of the power units to his/her legs. There are angular sensors and FRF sensors to measure motion information of HAL-5 Type-C and a wearer for wearer's intention estimation. Potentiometers as angular sensors are attached to the each joint to measure the joint angles. FRF sensors utilizing the semiconductor-type pressure sensor are implemented in shoes. Figure 4 shows the appearance of the shoes of HAL- 5 Type-C with built-in FRF sensors. The weight of a wearer including HAL-5 Type-C is transferred onto the 
sensor unit and measured by the pressure sensors. These sensors can also measure the distribution of load between a toe part and a heel part during walking and standing because two sensors are built in the front and rear of the shoe sole inside. In addition, a computer and batteries are attached on a wearer's waist, and motor drivers and other electrical circuits for the signal processing are allocated on each power unit. Compared with the robot suit HAL-3 (see Fig. 1(b)), HAL-5 Type-C is improved for patients' daily use since there is no large backpack on his/her back and a width of the power units in the back view becomes thin enough to pass through narrow spaces as shown in Fig. 2. Figure 5 shows angles and rotation directions of each joint described in this paper.

\section{CONTROLLER DESIGN}

In this section, we explain a controller for walking support system. Walking motion in this work shall be consist of three functions including swinging a leg, landing and supporting a body as shown in Fig. 6. In this paper, we call each span of three functions "swing phase", "landing phase" and "support phase". In the swing phase, the patterns extracted from healthy person's walk are applied as the reference patterns of the proportional and derivative (PD) control for the corresponding joints of a wearer. The reference patterns are used for the corresponding leg's control synchronizing with wearer's intention estimated by our proposed algorithm. In the landing phase, we realize the leg function for a foot landing by not tracking reference patterns but applying constant-value control. Based on our conventional work [12], we found that the knee joint of a wearer at landing instance is apt to be flexed by his/her own weight and much torque beyond the torque tolerance is needed to compensate for the knee bend. Therefore the knee joint has to be extended earlier than the reference pattern by constant-value control. In the support phase as well as the landing phase, the leg is supported by constant-value control in order to support his weight by one leg. The following sub-sections explain the details of the controller algorithm.

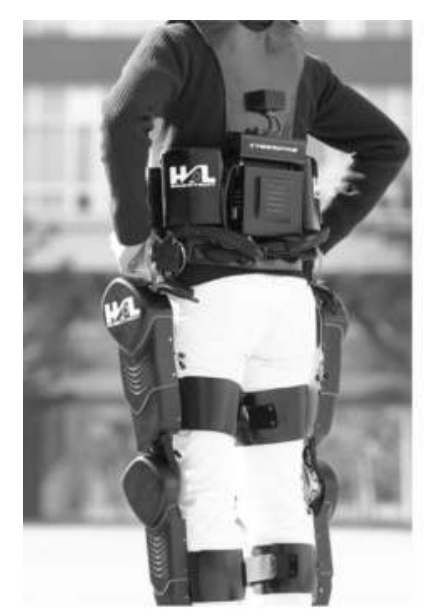

(a) Back view.

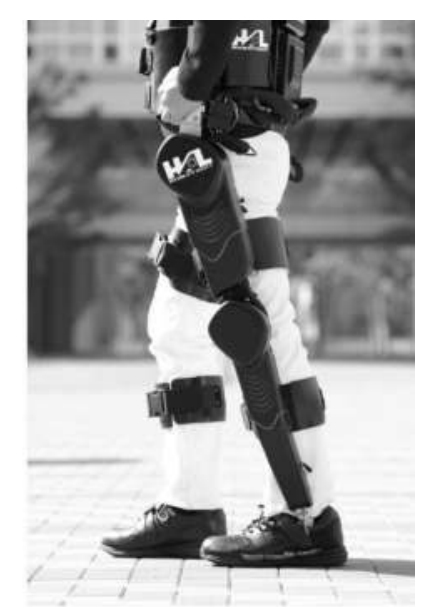

(b) Side view.

Fig. 2. HAL-5 Type-C developed for walking support of a paraplegia patient. Total weight is $15 \mathrm{~kg}$. 


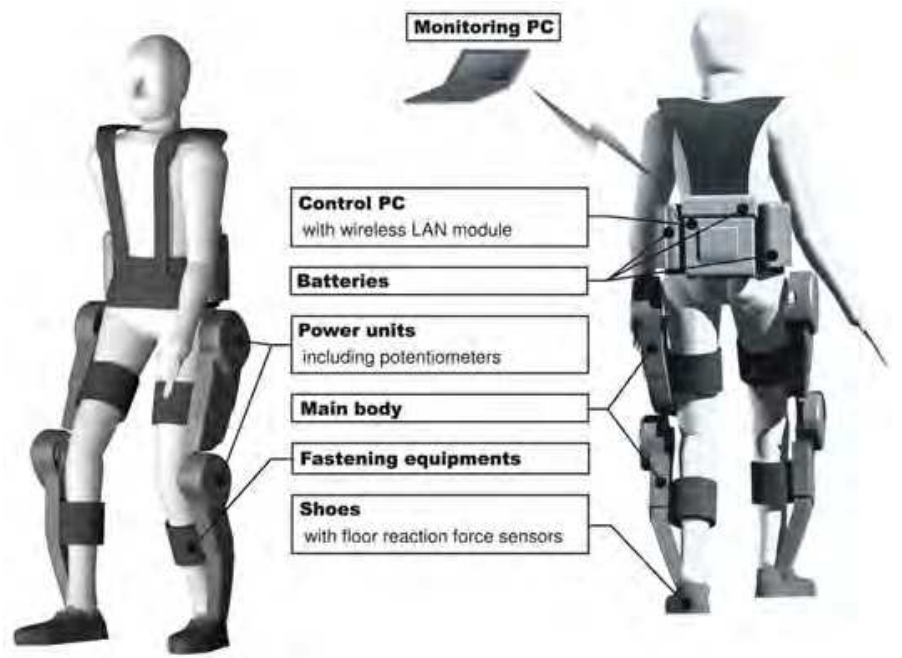

Fig. 3. System configurations of HAL-5 Type-C.

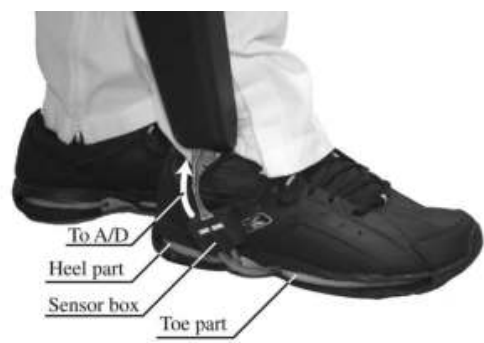

Fig. 4. Built-in floor reaction force sensors.

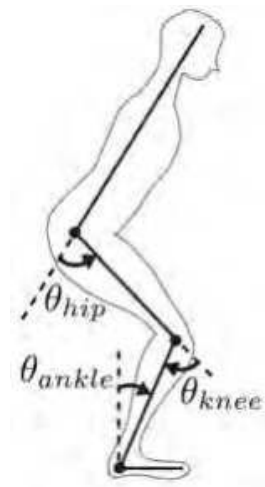

Fig. 5. Rotation directions of each joint. 


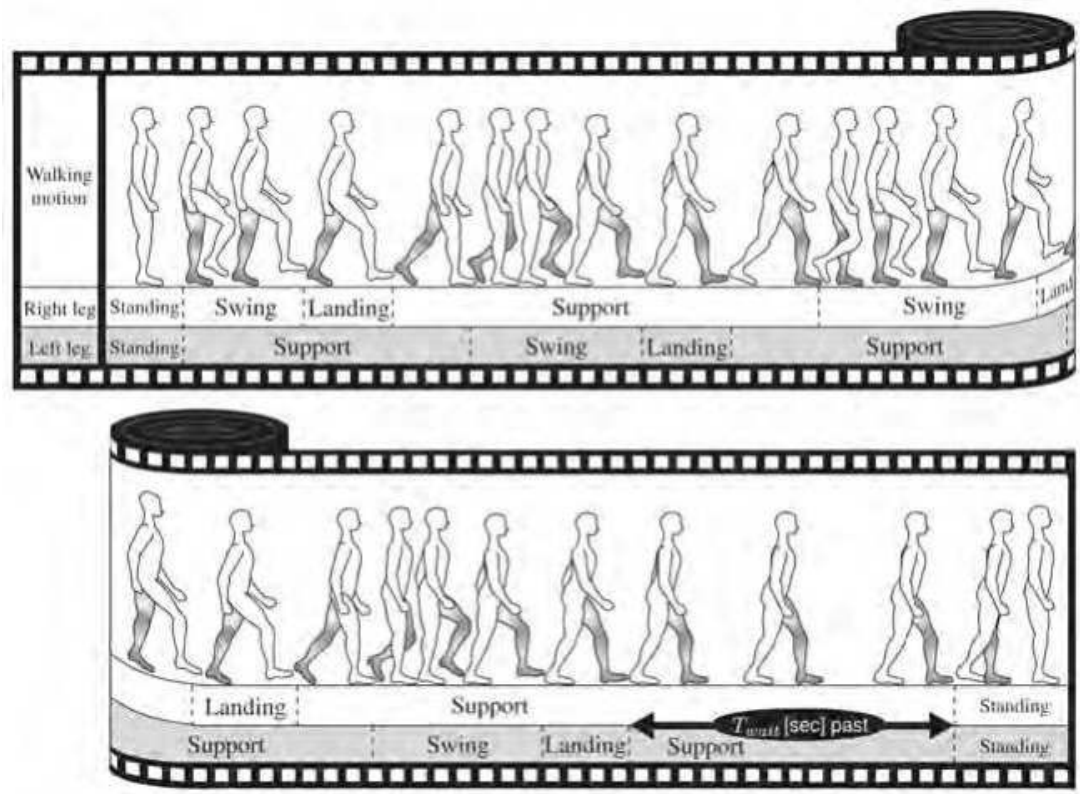

Fig. 6. Three functions in walking motion.

\subsection{Reference pattern generation}

As mentioned above, a swing leg in the swing phase is supported by applying reference walking patterns measured in healthy person's walk. The reference patterns are generated in the following process.

1. To measure angle data of hip and knee joints in healthy person's walk.

2. To divide a sequence of the measured walk pattern into patterns of each step and then average the walk patterns.

3. To divide the averaged pattern into three phases and extract a pattern in the swing phase.

At first, we measure a healthy person's walk to acquire the angle data of hip and knee joints during walk. In this experiment, we measure a normal walk of a man in his twenties, who has the similar body constitutions including height, weight and length of legs to the subject A. Second, a sequence of the measured walk pattern is divided into patterns in each step and then they are averaged. At this stage, we should pay attention that habits of walking and asymmetry between right and left leg step are not reflected in the extracted patterns strongly. Figure 7(a) shows walking patterns in one step averaged in this experiment.

Finally, the averaged walking patterns are divided into patterns in the swing, landing and support phase. The swing phase is between a moment when a foot leaves a floor and a moment when a thigh is full flexed. The landing phase continues until a moment when a foot of the swing leg contacts a ground, and the support phase continues until a moment when one step finishes. The walking patterns extracted from a healthy person's walk are shown in Fig. 7(b), (c) and (d). Namely, Fig. 7(b) shows the reference angle patterns in the swing phase 


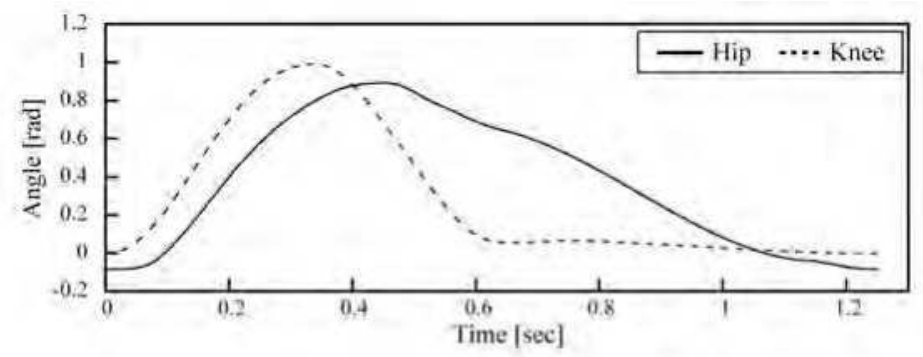

(a)

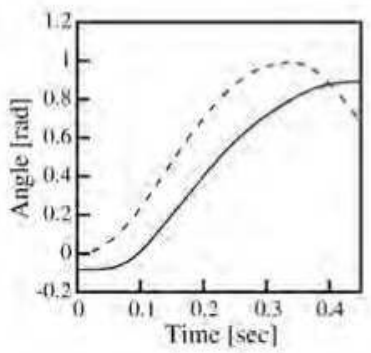

(b)

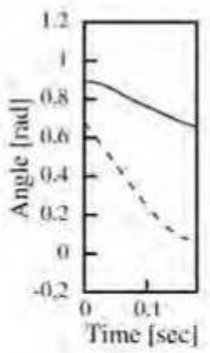

(c)

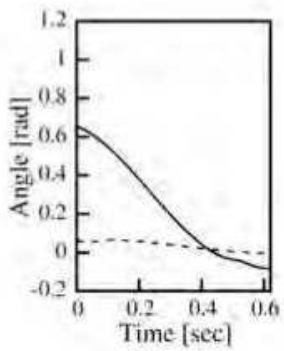

(d)

Fig. 7. Reference walking patterns of joint angle. (a) Patterns in one cycle of walk. (b) Patterns in the swing phase. (c) Patterns in the landing phase. (d) Patterns in the support phase.

used in this walking support. The PD controller to drive a leg swing needs reference angular velocity patterns as well as the angle patterns, and the angular velocity patterns are generated by differentiating the angle patterns with respect to time. In addition, the time scales of the reference patterns are linearly shorten or lengthen so that the walking cycle could be adjusted to a wearer's intentions or a wearer's body constitutions.

On the other hand, a swing leg in the landing phase is supported by constant-value control for the preparation of patient's weight support. The reference angle and angular velocity in the landing and support phase are empirically set. Table 1 shows reference values in each phase of walking support. In this table, $\theta_{\text {href }}$ and $\dot{\theta}_{\text {href }}$ show the reference angle and angular velocity of a hip joint respectively, and $\theta_{\text {kref }}$ and $\dot{\theta}_{\text {kref }}$ show the reference angle and angular velocity of a knee joint respectively. In addition, the hip and knee joints should be straightened through the landing and support phase in order to support a wearer's weight by one leg. Therefore, the reference angle of hip joint in the landing phase is 0 rad. Table 1, however, shows the reference angle of knee joint is not 0 rad but $-0.052 \mathrm{rad}$. This over extension of the knee joint can prevent the knee joint from bending due to an impact of landing a foot and gravity. In general, it is quite harmful for human to extend the knee joint excessively, but HAL does not extend wearer's joints beyond the range of motion since fastening equipments of HAL made of rigid plastic has a little flexibility and mechanical limiters at knee joints prevent the joints from extending more than that angle. HAL controls the joint angle to keep the reference values in the support phase until the end of the single leg support phase when a foot of an opposite side swing leg touches on a floor. After the foot of the swing leg makes a contact with a floor, 


\begin{tabular}{cccc}
\hline & Swing phase & Landing phase & Support phase \\
\hline$\theta_{\text {href }}[\mathrm{rad}]$ & Fig. 7(b) & 0.0 & $0.0(-0.7)$ \\
$\dot{\theta}_{\text {href }}[\mathrm{rad} / \mathrm{sec}]$ & Time derivative of Fig. 7(b) & 0.0 & 0.0 \\
$\theta_{\text {kref }}[\mathrm{rad}]$ & Fig. 7(b) & -0.052 & -0.052 \\
$\dot{\theta}_{\text {kref }}[\mathrm{rad} / \mathrm{sec}]$ & Time derivative of Fig. 7(b) & 0.0 & 0.0 \\
\hline
\end{tabular}

Table 1 . Reference values in one cycle of walking support.

the reference hip joint angle of the supporting leg switches from $0.0 \mathrm{rad}$ to $-0.7 \mathrm{rad}$ shown in parentheses of Table 1. This hip extension contributes to the smooth weight shift from a current supporting leg to a following one. Reference angular velocity of both joints in the landing and support phase consistently maintains $0.0 \mathrm{rad} / \mathrm{sec}$ through one cycle of walking support.

\subsection{Intention estimator}

Estimation of patients' intentions contributes to support not only comfortably but also safely, because an inconformity between the robot suit motion and the patient motion results in his stumbling or falling. Instead of the bioelectrical signals used for the control of the conventional HAL, the floor reaction force is used for an intention estimation of the subject A who can control his weight balance using two canes with his hands. The floor reaction force reflects the position of center of gravity (COG) and COG could be the reliable information for the intention estimation. For example, a leg could leave a floor and work as a swing leg safely if it does not support his/her weight. A support system "HAL" estimates which leg supports a wearer's weight, when a wearer begins to swing a right or left leg and when he/she wants to stop walking. At first, for example, a right leg is considered to be a support leg when a foot contact condition:

$$
\begin{gathered}
f_{r h}>\alpha_{r h} \quad \text { or } \\
f_{r t}>\alpha_{r t}
\end{gathered}
$$

is satisfied, where $f_{r h}$ and $f_{r t}$ are FRF of a right foot heel side and toe side, respectively. In addition, $\alpha_{r h}$ and $\alpha_{r t}$ are thresholds to detect a landing of a right foot. In general, the condition (1) is applied in advance of (2) since a healthy person puts a heel of a swing leg on a floor in advance of a toe. Patients with paralysis on legs such as the subject A, however, have a foot weighed down and may put a toe of a swing leg on a floor in advance of a heel. The condition (2) is effective in detecting the landing in cases of paraplegia patients. On the other hand, a left leg is considered to be a support leg when a foot contact condition:

$$
\begin{gathered}
f_{l h}>\alpha_{l h} \text { or } \\
f_{l t}>\alpha_{l t}
\end{gathered}
$$

is satisfied, where $f_{l h}$ and $f_{l t}$ are FRF of a left foot heel side and toe side, respectively. In addition, $\alpha_{l h}$ and $\alpha_{l t}$ are thresholds to detect a landing of a left foot.

Second, for example, HAL estimates the intention that a wearer wants to swing a right leg when swing start conditions:

$$
f_{r h}<\beta_{r h} \text { and }
$$




$$
f_{r t}<\beta_{r t}
$$

are satisfied, where $\beta_{r h}$ and $\beta_{r t}$ are thresholds to detect a moment when each part of a right foot leaves a floor. On the other hand, HAL estimates the intention that a wearer wants to swing a left leg when swing start conditions:

$$
\begin{gathered}
f_{l h}<\beta_{l h} \text { and } \\
f_{l t}<\beta_{l t}
\end{gathered}
$$

are satisfied, where $\beta_{l h}$ and $\beta_{l t}$ are thresholds to detect a moment when each part of a left foot leaves a floor. In this study, the following two constraint conditions are added to the above conditions for more stable estimation of wearer's intentions.

1. Do not start to swing a leg unless a foot of the opposite side leg is on a floor.

2. Do not swing the same leg sequentially.

HAL estimates the intention that a wearer wants to stop in his/her tracks if it pasts a certain time before the swing start conditions (5) and (6), or (7) and (8) are satisfied. In the walking support, HAL stops the sequential walking supports and helps a wearer come back to the standing posture when a condition:

$$
\begin{gathered}
t_{\text {cur }}-t_{r}>T_{\text {wait }} \text { or } \\
t_{\text {cur }}-t_{l}>T_{\text {wait }}
\end{gathered}
$$

is satisfied, where $t_{c u r}, t_{r}$ and $t_{l}$ are the current time and the time when the last right or left foot touches on a floor. In addition, $T_{\text {wait }}$ is a temporal threshold to switch the walking support to the standing posture support. In this moment, the reference angles of all joints are almost zero, therefore a backward leg is replaced around a forward leg if a load on the backward leg becomes almost zero by his/her weight shift. We set $T_{\text {wait }}=5.0 \mathrm{sec}$ in this experiment.

\subsection{Control Architecture}

Bipedal locomotion using patient's legs is achieved by the tracking control and by phase synchronization of motion support with patient's intention. This control consists of the PD control using reference walking patterns based on healthy person's walk as shown in Fig. 7(a) in the swing phase and the constant-value control in the landing and support phase. Figure 8 shows a block diagram for this tracking control and phase synchronization. The human intention estimator (HIE) located in the upper-left part in the figure has the FRF as inputs for the estimation algorithms described in the section 4.2. Three blocks under the HIE are a library of the reference patterns in the swing phase and the reference values in the landing and support phase. The HIE allocates these references to two legs during walking. There are six ordinary PD control blocks on the right side of the HIE and the library. The upper three blocks are controllers for the right leg and the lower ones are for the left leg. The command voltages $\tau_{r}$ and $\tau_{l}$ to the power units on both legs are calculated by:

$$
\boldsymbol{\tau}_{r}=\boldsymbol{K}_{r}\left(\boldsymbol{C}_{r} \boldsymbol{\theta}_{r e f}-\boldsymbol{\theta}_{r}\right)+\hat{\boldsymbol{K}}_{r}\left(\boldsymbol{C}_{r} \dot{\boldsymbol{\theta}}_{\boldsymbol{r e f}}-\dot{\boldsymbol{\theta}}_{r}\right) \text { and }
$$




$$
\boldsymbol{\tau}_{l}=\boldsymbol{K}_{l}\left(\boldsymbol{C}_{l} \boldsymbol{\theta}_{r e f}-\boldsymbol{\theta}_{l}\right)+\hat{\boldsymbol{K}}_{l}\left(\boldsymbol{C}_{l} \dot{\boldsymbol{\theta}}_{r e f}-\dot{\boldsymbol{\theta}}_{l}\right),
$$

where $\boldsymbol{\theta}_{r}$ and $\boldsymbol{\theta}_{l}$ are the actual wearer's leg joint angles, $\dot{\boldsymbol{\theta}}_{r}$ and $\dot{\boldsymbol{\theta}}_{l}$ are angular velocities and subscripts $r$ and $l$ mean right and left, respectively. In addition, $\boldsymbol{\theta}_{r e f}$ and $\dot{\boldsymbol{\theta}}_{r e f}$ are the reference joint angles and the reference angular velocities, respectively. These variables including $\tau_{r}$ and $\boldsymbol{\tau}_{l}$ have two elements that correspond to two joints: hip and knee joint. $\boldsymbol{\tau}_{r}, \boldsymbol{\tau}_{l}, \boldsymbol{\theta}_{r}, \boldsymbol{\theta}_{l}, \dot{\boldsymbol{\theta}}_{r}, \dot{\boldsymbol{\theta}}_{l}, \boldsymbol{\theta}_{r e f}$ and $\dot{\boldsymbol{\theta}}_{\text {ref }}$ are given as follows:

$$
\begin{gathered}
\boldsymbol{\tau}_{r}=\left[\begin{array}{c}
\tau_{r h} \\
\tau_{r k}
\end{array}\right], \quad \boldsymbol{\tau}_{l}=\left[\begin{array}{c}
\tau_{l h} \\
\tau_{l k}
\end{array}\right], \\
\boldsymbol{\theta}_{r}=\left[\begin{array}{c}
\theta_{r h} \\
\theta_{r k}
\end{array}\right], \quad \boldsymbol{\theta}_{l}=\left[\begin{array}{c}
\theta_{l h} \\
\theta_{l k}
\end{array}\right], \quad \dot{\boldsymbol{\theta}}_{r}=\left[\begin{array}{c}
\dot{\theta}_{r h} \\
\dot{\theta}_{r k}
\end{array}\right], \quad \dot{\boldsymbol{\theta}}_{l}=\left[\begin{array}{c}
\dot{\theta}_{l h} \\
\dot{\theta}_{l k}
\end{array}\right], \\
\boldsymbol{\theta}_{r e f}=\left[\begin{array}{c}
\theta_{\text {href }} \\
\theta_{k r e f}
\end{array}\right], \quad \dot{\boldsymbol{\theta}}_{r e f}=\left[\begin{array}{c}
\dot{\theta}_{h r e f} \\
\dot{\theta}_{k r e f}
\end{array}\right],
\end{gathered}
$$

where subscripts $r h, r k, l h$ and $l k$ mean right hip joint, right knee joint, left hip joint and left knee joint, respectively. On the other hand, $\boldsymbol{K}_{r}$ and $\boldsymbol{K}_{l}$ are feedback gains of the joint angle errors, and $\hat{\boldsymbol{K}}_{r}$ and $\hat{\boldsymbol{K}}_{l}$ are feedback gains of the joint angular velocity errors. The different feedback gains are used in the swing, landing or support phase independently by adopting this control architecture. In addition, $\boldsymbol{C}_{r}$ and $\boldsymbol{C}_{l}$ are gains to the reference joint angles and angular velocities. These gains can adjust a joint flexion and a stride length in a wearer's supported walk. In this experiment, we set $\boldsymbol{C}_{l}$ larger than $\boldsymbol{C}_{r}$ in order to avoid collisions of a left leg which has a more severe paralysis with a floor in the swing phase. $\boldsymbol{K}_{r}, \boldsymbol{K}_{l}, \hat{\boldsymbol{K}}_{r}, \hat{\boldsymbol{K}}_{l}, \boldsymbol{C}_{r}$ and $\boldsymbol{C}_{l}$ are diagonal matrixes which are given as follows:

$$
\begin{array}{cc}
\boldsymbol{K}_{r}=\left[\begin{array}{cc}
k_{r h} & 0 \\
0 & k_{r k}
\end{array}\right], \quad \boldsymbol{K}_{l}=\left[\begin{array}{cc}
k_{l h} & 0 \\
0 & k_{l k}
\end{array}\right], \quad \hat{\boldsymbol{K}}_{r}=\left[\begin{array}{cc}
\hat{k}_{r h} & 0 \\
0 & \hat{k}_{r k}
\end{array}\right], \quad \hat{\boldsymbol{K}}_{l}=\left[\begin{array}{cc}
\hat{k}_{l h} & 0 \\
0 & \hat{k}_{l k}
\end{array}\right], \\
\boldsymbol{C}_{r}=\left[\begin{array}{cc}
c_{r h} & 0 \\
0 & c_{r k}
\end{array}\right], \quad \boldsymbol{C}_{l}=\left[\begin{array}{cc}
c_{l h} & 0 \\
0 & c_{l k}
\end{array}\right] .
\end{array}
$$

Moreover, the PD gains of swing leg control $k_{r h}, k_{l h}, \hat{k}_{r h}, \hat{k}_{l h}, k_{r k}, k_{l k}, \hat{k}_{r k}$ and $\hat{k}_{l k}$ were determined based on frequency responses and step responses of hip and knee joints. The concrete procedure is described in Appendix A.

The control flow for the walking support is as follows. At first, HAL supports a wearer's standing posture. Once the conditions shown in the equations (5) and (6) are satisfied, HAL starts the PD control for the swing phase in a right leg and for the support phase in a left leg. On the other hand, HAL starts the PD control for the swing phase in a left leg and the support phase in a right leg once the conditions shown in the equations (7) and (8) are satisfied. The PD control for a swing leg continues until HAL finishes the reference swing patterns. After that, HAL runs the constant-value control for the landing phase until the condition shown in the equation (1) or (2) is satisfied in a case of a right leg and until the condition shown in the equation (3) or (4) is satisfied in a case of a left leg. The other leg continues the control for the support phase. After HAL detects a contact between a foot of a swing leg and a floor, HAL runs the constant-value control for the support phase on both legs and continues the control until the next swing start conditions are satisfied. If the conditions are not satisfied, 


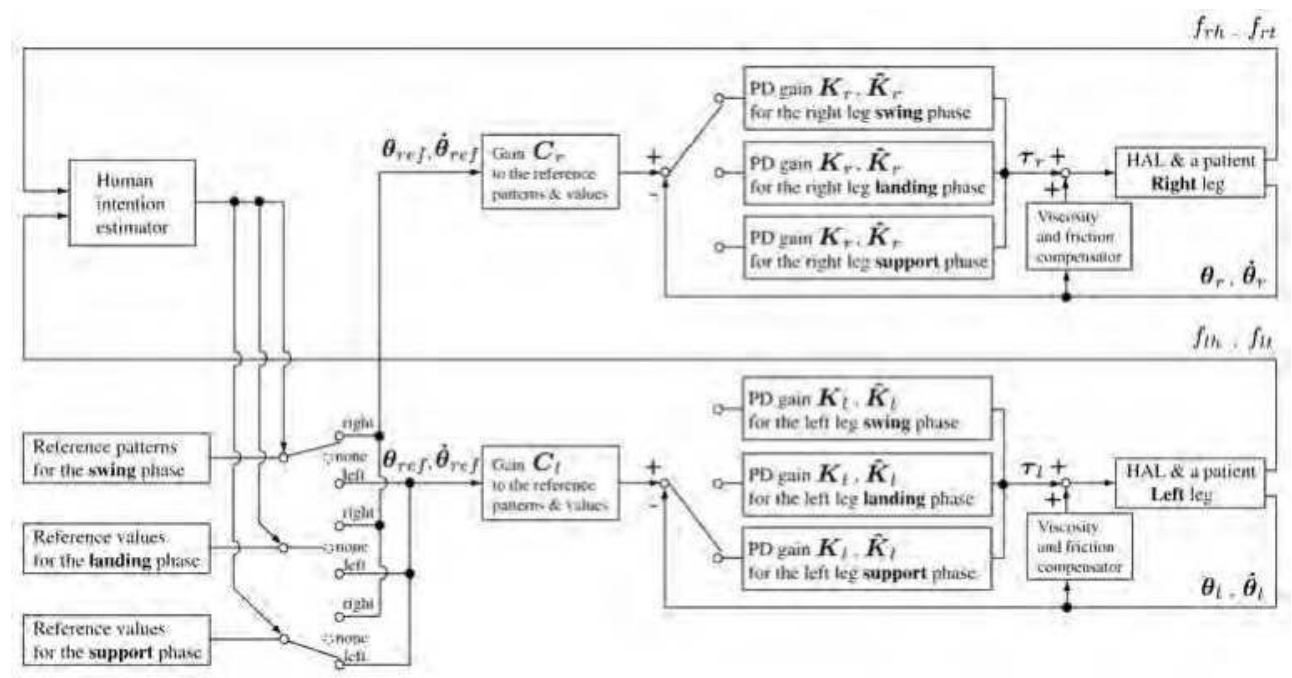

Fig. 8. Block diagram for tracking control.

two legs are kept at the final posture of the step. However, the reference angles of all joints are almost zero in this phase, therefore a backward leg is replaced around a forward leg if a load on the backward leg becomes almost zero by his/her weight shift. Thus, a wearer can come back to the standing posture. This algorithm can synchronize walking support with human intentions at a walk start instance, a walk stop instance as well as the beginning of leg swing during walking. In addition to those walking support, HAL compensates viscosity and static friction of the power units [3].

\section{EXPERIMENT}

The subject $A$ is the patient who has a strong sensory paralysis especially on the left leg and can walk slowly using two canes with his both hands. Since he can stand by himself, the support aim with HAL is to help his leg swinging forward and sustaining his weight $(65 \mathrm{~kg})$. This support contributes to stabilize his walk by pushing a swing leg forward and by avoiding collisions of a swing leg with a floor. In this experiment, the patient is supposed to keep his own stability by holding a walking frame with his arms and a staff supports the walking frame for the sake of the patient's safety as shown in Fig. 9.

\subsection{Experimental setup}

In this experiment, the whole thresholds to detect a moment when a foot leaves a floor or contacts on a floor expressed as $\alpha_{r h}, \alpha_{r t}, \alpha_{l h}, \alpha_{l t}, \beta_{r h}, \beta_{r t}, \beta_{l h}$ and $\beta_{l t}$ are finally set to $50 \mathrm{~N}$ based on the subject's weight and his impression after some trials. On the other hand, the feedback gains for the joint control $k_{r h}, k_{l h}, \hat{k}_{r h}, \hat{k}_{l h}, k_{r k}, k_{l k}, \hat{k}_{r k}$ and $\hat{k}_{l k}$, the gains to the reference joint angle and velocity errors $c_{r h}, c_{r k}, c_{l h}$ and $c_{l k}$ and a time span for swinging a leg are adjusted through some trials reflecting the subject's impression. The time span for swinging a leg is finally set to $0.9 \mathrm{sec}$ at the time. 


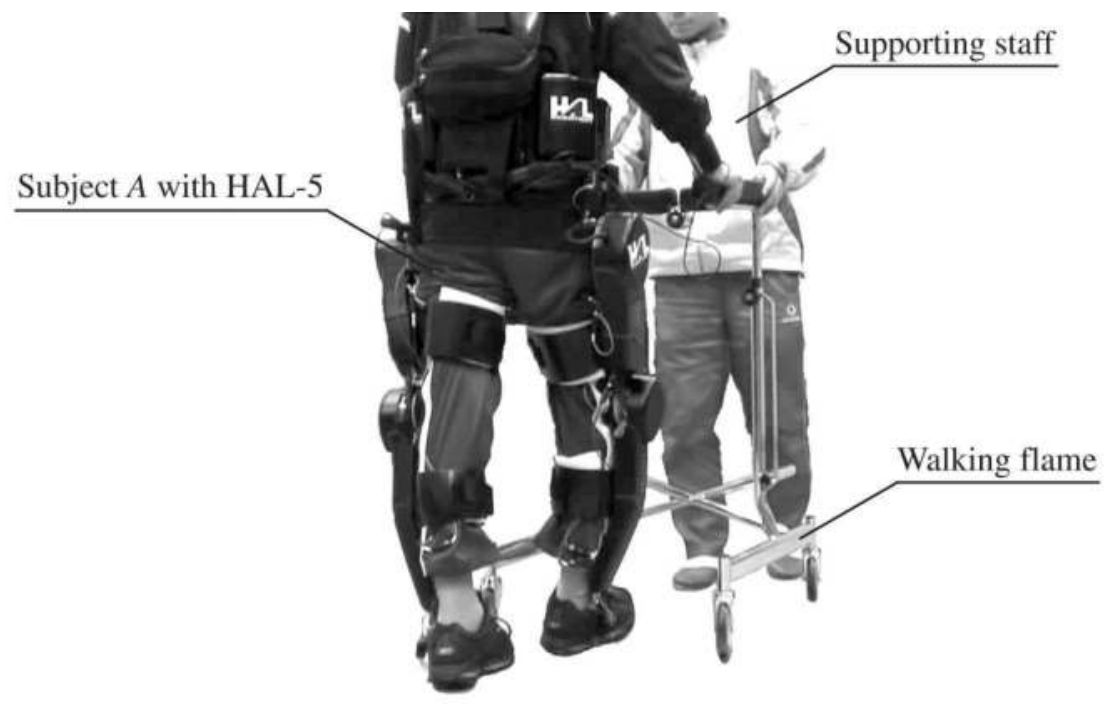

Fig. 9. Experimental setting.

\subsection{Results}

Figures 10 and 11 show the FRF data and phase transitions on each leg during a walking support. In both figures, one leg performs as the support leg up to a toe-off moment when the equation (6) or (8) is satisfied and then the leg performs as the swing leg for $0.9 \mathrm{sec}$ and the leg begins to support his weight as the support leg from a heel-on moment when the equation (1) or (3) is satisfied shortly after the start of the landing phase. In addition, Fig. 12 shows the FRF data on both legs and the phase transitions at the start of walking support. The FRF of the heel part is almost zero since the subject A leans on the walking frame for the sake of safety. On the other hand, the FRF of the toe part reflects the shift of his COG. At first, he stands on his legs with a load distribution which the right leg supports about $250 \mathrm{~N}$ and the left leg supports about $350 \mathrm{~N}$. After that, he shifts his COG in a direction toward his left side, and finally the right and left leg begins to perform as a swing leg and support leg, respectively when the equations (5) and (6) are satisfied. HAL starts supporting the walk of the subject A synchronizing his intentions. Figures 13 and 14 show his each joint angles, their references and torques of the power units during walking support. From the results of joint angles in these figures, his hip and knee joints follow the reference angles in a almost part of time in one cycle of the supported walk. HAL supports his walk based on a healthy person's walk as shown in Fig. 7. On the other hand, the results in a latter part of the swing phases show his joints do not follow the references, especially knee joint on his left leg which has a severe sensory trouble. The knee joint of the subject A resists the actuator of HAL since he does not get used to receiving the physical support. The tracking error will be small after enough training for relaxation of the knee joint in the swing phase. 


\section{CONCLUSIONS}

In this chapter, we have proposed the algorithm to estimate patients' intentions so that the HAL-5 Type-C could support a patient with paraplegia to walk. The estimation algorithm based on the floor reaction force was investigated through the walking support experiments for a patient with a sensory paralysis on both legs. The cycle of reference walking patterns was adjusted for the patient and the walking support based on the reference walking was achieved, synchronizing with a patient's intentions estimated by the algorithm. We confirmed that the algorithm successfully estimated corresponding to a patient's intentions. However, it did not stabilize a patient's body posture and he had to maintain his balance using a walking frame with his hands. One of our future works is to develop a stabilizing algorithm and mechanism so that his hand regains its own functions. 


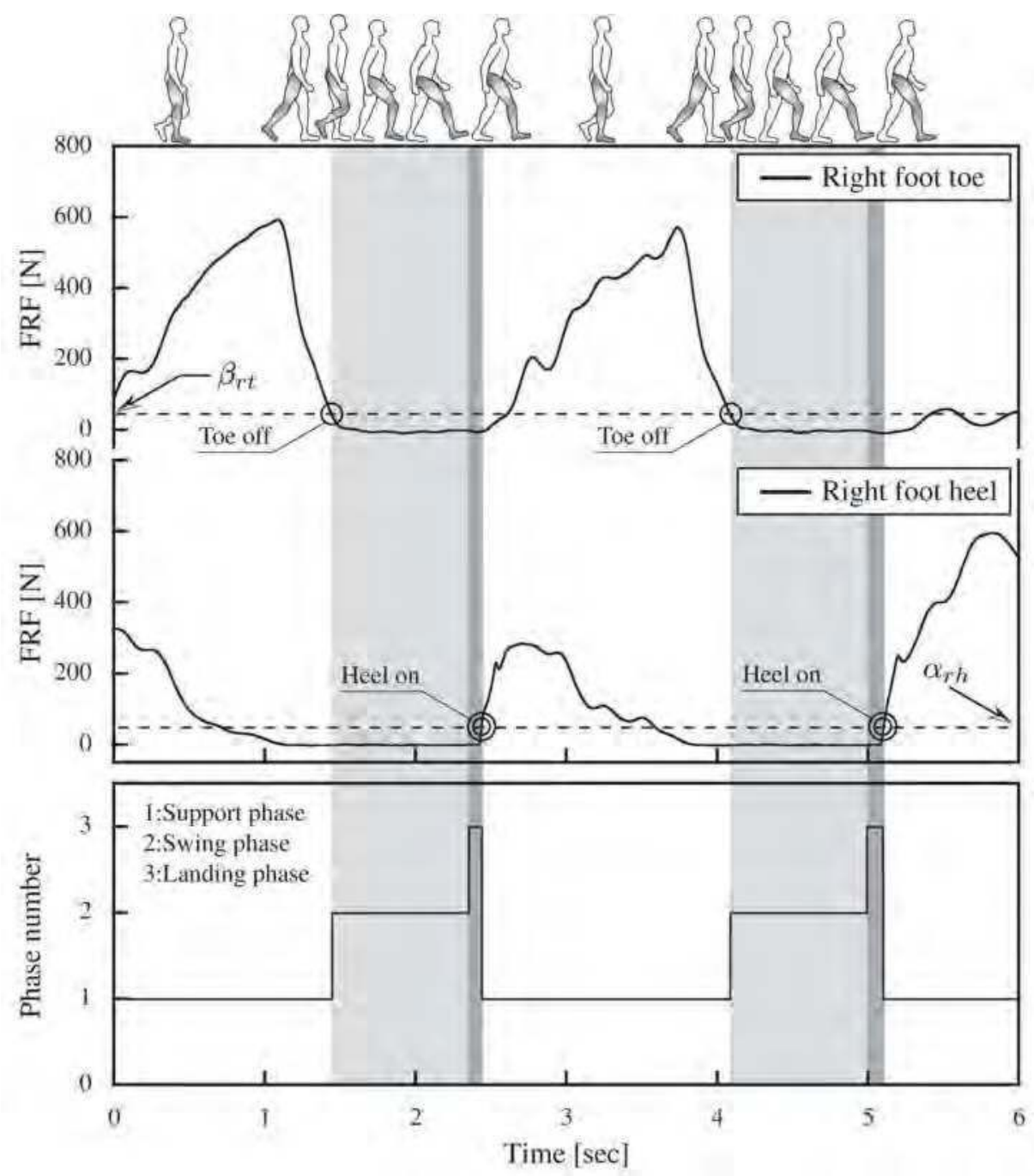

Fig. 10. Result of FRF-based intention estimation on right leg. 


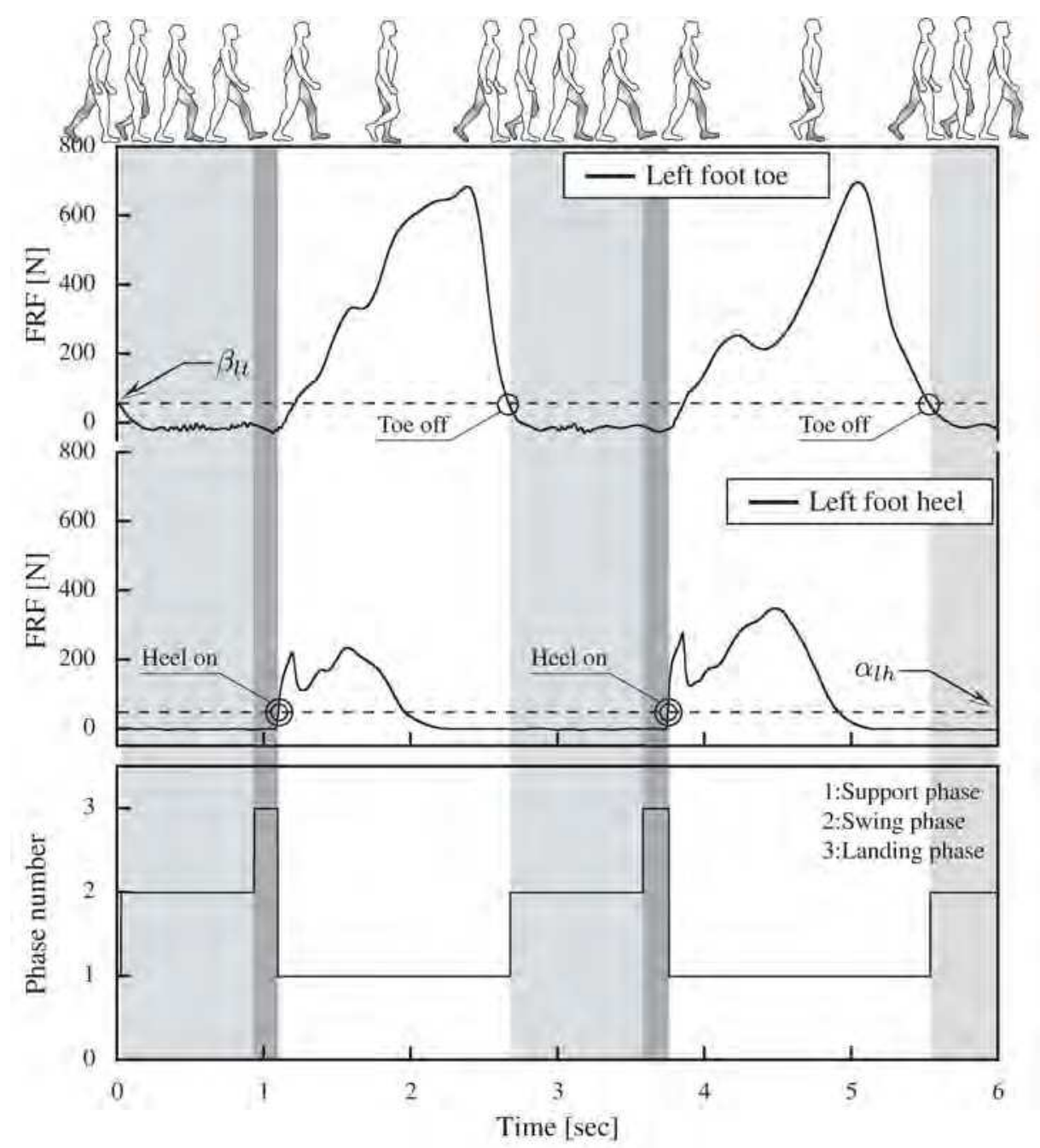

Fig. 11. Result of FRF-based intention estimation on left leg. 


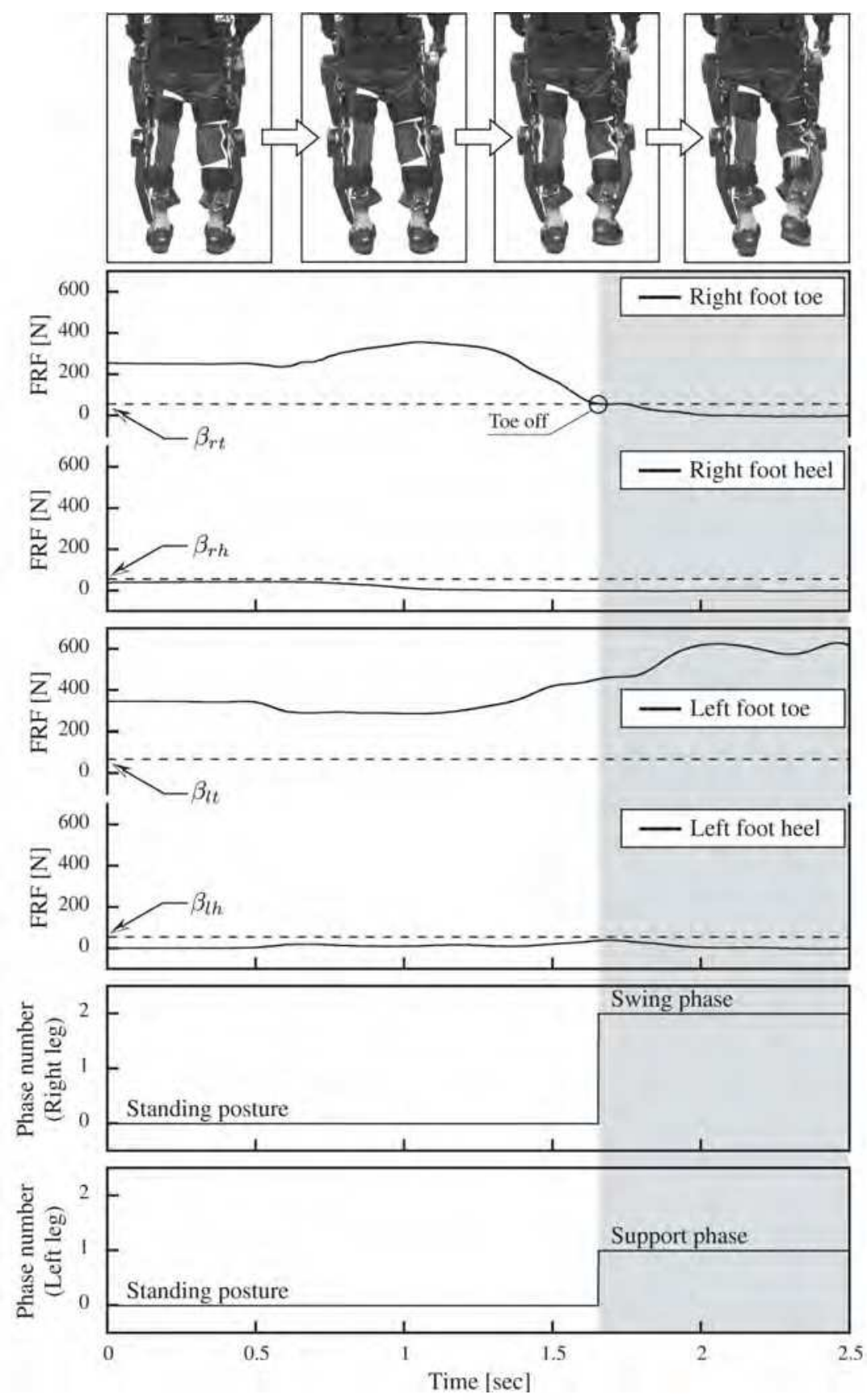

Fig. 12. Start of walking support by intention estimation. 


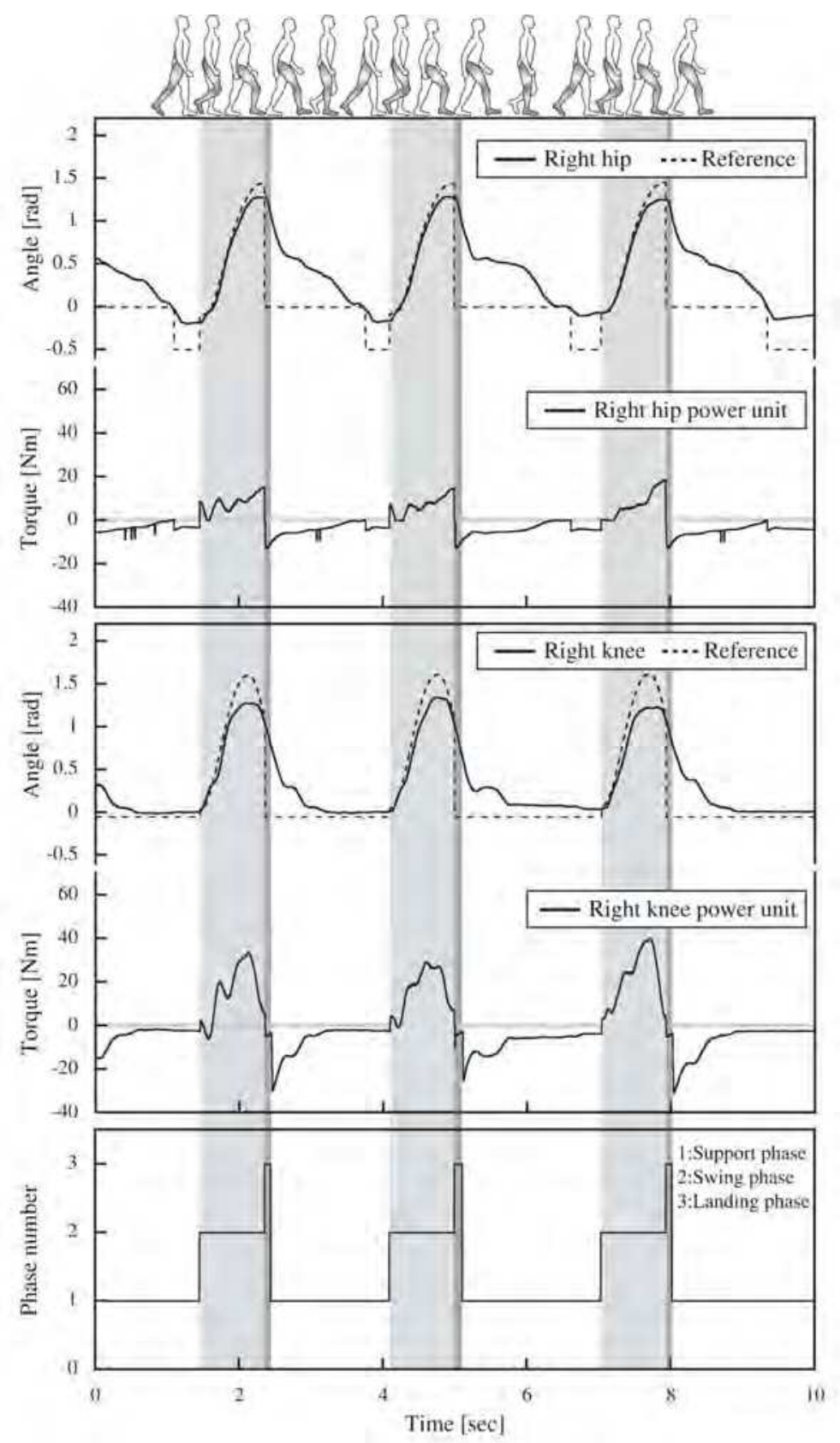

Fig. 13. Right leg joint angles with reference angles and power units' torques in each phase. 


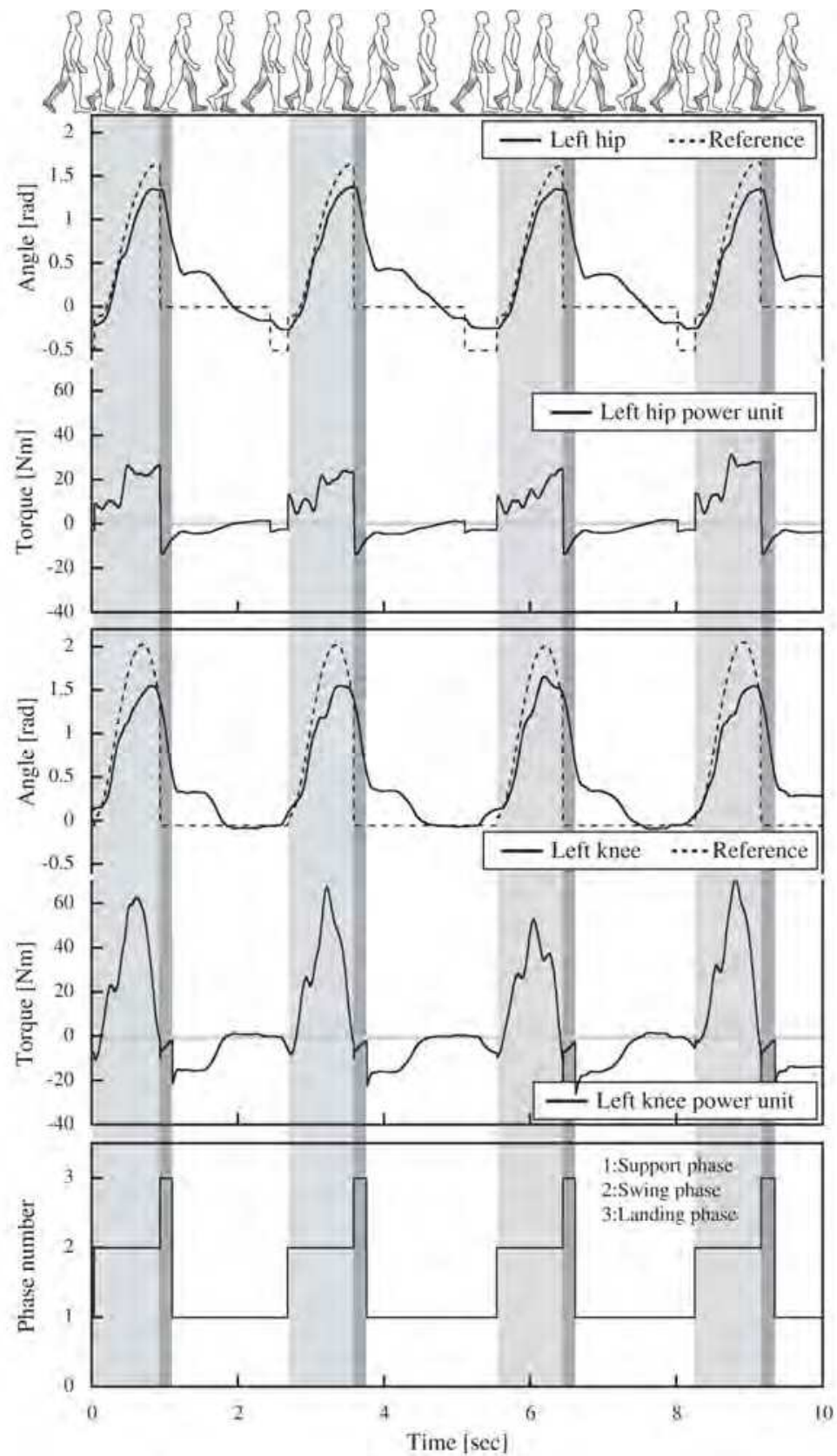

Fig. 14. Left leg joint angles with reference angles and power units' torques in each phase. 


\section{Acknowledgments}

This research was partially supported by Grant-in-Aid for Scientific Research (General Research (A), No. 17206045, 2005-2008) from the Ministry of Education, Culture, Sports, Science and Technology of Japan and by Health and Labour Sciences Research Grants (No. H17-Physi005, 2005-2007) from the Ministry of Health, Labour and Welfare of Japan.

\section{REFERENCES}

[1] Junpei Okamura, Hiroshi Tanaka and Yoshiyuki Sankai, EMG-based Prototype Powered Assistive System for Walking Aid, in Proc. of Asian Symposium on Industrial Automation and Robotics (ASIAR'99), Bangkok, pp.229-234 (1999).

[2] Takao Nakai, Suwoong Lee, Hiroaki Kawamoto and Yoshiyuki Sankai, Development of Powered Assistive Leg for Walking Aid using EMG and Linux, in Proc. of Asian Symposium on Industrial Automation and Robotics (ASIAR'01), Bangkok, pp.295-299 (2001).

[3] Lee S. and Sankai Y., Power Assist Control for Walking Aid with HAL-3 Based on EMG and Impedance Adjustment around Knee Joint, Proc. of IEEE/RSJ International Conference on Intelligent Robots and Systems (IROS 2002), EPFL, Switzerland, pp.1499-1504 (2002).

[4] Jerry E. Pratt, Benjamin T. Krupp, Christopher J. Morse and Steven H. Collins, The RoboKnee: An Exoskeleton for Enhancing Strength and Endurance During Walking, in Proc. of the 2004 IEEE International Conference on Robotics and Automation (ICRA '04), New Orleans, pp.2430-2435 (2004).

[5] Takahiko Nakamura, Kazunari Saito and Kazuhiro Kosuge, Control of Wearable Walking Support System Based on Human-Model and GRF, Proc. of the 2005 IEEE International Conference on Robotics and Automation (ICRA '05), Barcelona, pp. 4405-4410 (2005).

[6] H. Kazerooni, Ryan Steger and Lihua Huang, Hybrid Control of the Berkeley Lower Extremity Exoskeleton (BLEEX), The International Journal of Robotics Research, vol. 25, pp. 561-573 (2006).

[7] Ryan Steger, Sung Hoon Kim and H. Kazerooni, Control Scheme and Networked Control Architecture for the Berkeley Lower Extremity Exoskeleton (BLEEX), in Proc. of the 2006 IEEE International Conference on Robotics and Automation (ICRA '06), Orlando, Florida, pp. 3469-3476 (2006).

[8] Hiroaki Kawamoto and Yoshiyuki Sankai, Power Assist System HAL-3 for Gait Disorder Person, Proc. of the 2002 International Conference on Computers Helping People with Special Needs (IC-CHP 2002), Linz, Austria, pp. 196-203 (2002).

[9] Hiroaki Kawamoto and Yoshiyuki Sankai, Power assist method based on Phase Sequence and muscle force condition for HAL, Advanced Robotics, vol. 19, No. 7, pp. 717-734 (2005).

[10] Masashi SATO, Hidetaka IKEUCHI, Ryozo KATOH and Tadashi YAMASHITA, Experimental Analysis of Reaction Force and Motion of Center of Gravity during Human Gait Initiation (Characteristics of Transferring from Transient Phase to Steady-State Phase), Trans. JSME (Series C), Vol.59, No.566, pp.3101-3107 (1993). (in Japanese)

[11] H. Ikeuchi, K. Shinkoda, R. Katoh, M.Sato, T. Yamashita, Analysis of Human Transient Walking by Wavelet Transform, Proc. of The Third International Symposium on Artificial Life and Robotics, Beppu, Japan, pp.695-698 (1998).

[12] Suzuki K., Kawamura Y., Hayashi T., Sakurai T., Hasegawa Y. and Sankai Y., IntentionBased Walking Support for Paraplegia Patient, Proc. of the 2005 IEEE International Conference on Systems, Man and Cybernetics (SMC2005), Hawaii, pp.2707-2713 (2005). 


\section{Appendix A}

The PD gains of swing leg control $k_{r h}, k_{l h}, \hat{k}_{r h}, \hat{k}_{l h}, k_{r k}, k_{l k}, \hat{k}_{r k}$ and $\hat{k}_{l k}$ (see equations (16)) were designed in preliminary experiments. In this appendix section, proportional gains for the hip joints $k_{r h}$ and $k_{l h}$ are expressed by $k_{* h}$, and proportional gains for the knee joints $k_{r k}$ and $k_{l k}$ are expressed by $k_{* k}$ for convenience of explanation. On the other hand, derivative gains for the hip joints $\hat{k}_{r h}$ and $\hat{k}_{l h}$ are expressed by $\hat{k}_{* h}$, and derivative gains for the knee joints $\hat{k}_{r k}$ and $\hat{k}_{l k}$ are expressed by $\hat{k}_{* k}$. Figure 15 shows experimental environments, where one leg can swing freely and the other leg supports a subject's weight. At first, hip or knee joint is controlled by the PD feedback control to examine the frequency response. The reference joint angle patterns are expressed by sine function with seven different frequencies, ranging from 0.1 to $3.0 \mathrm{~Hz}$, and with 1.0 rad peak-to-peak amplitude. Five different PD gains, ranging that the proportional gain is from 20.0 to 200.0 and the derivative gain is from 0.02 to 0.20 , are tested. Figures 16 and 18 show the frequency responses of hip and knee joints on Bode plot.

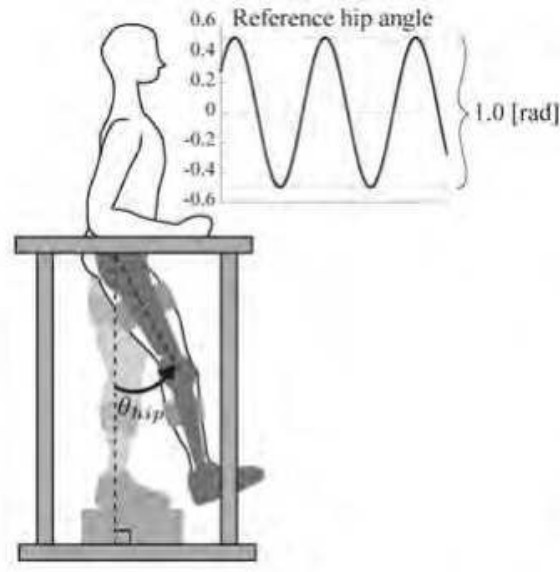

(a) Experimental motion for hip joint.

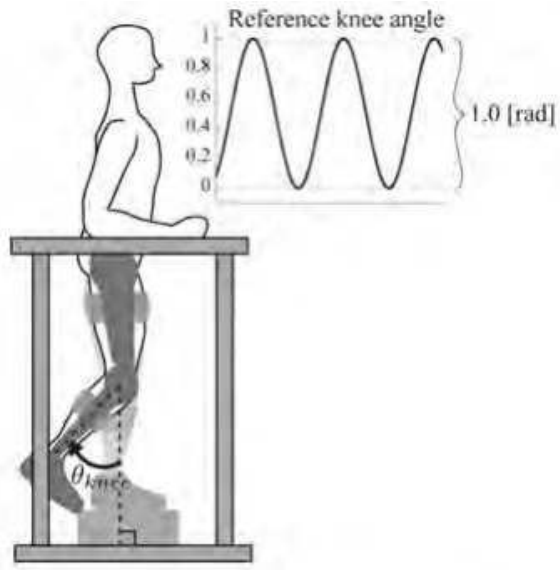

(b) Experimental motion for knee joint

Fig. 15. Experimental settings for each joint frequency response. A subject with the robot suit HAL stands inside a frame with one leg on a raised block so that the other leg can swing freely. The subject are asked to keep the upper body upright and completely relax the leg to follow reference joint motions produced by the HAL's power units. Two sine curves in the figure show the reference joint angle patterns on each joint.

Then, a unit step response of each joint is examined with six different PD gains. Figures 17 and 19 show the responses of hip and knee joints. From the viewpoint of amplitude characteristic, resonance frequency and phase shift on the hip joint shown in Fig. 16, the sufficient response could be obtained when the range that $k_{* h}$ was from 100.0 to 200.0 and $\hat{k}_{* h}$ was from 0.10 to 0.20 at less than $0.5 \mathrm{~Hz}$, which equaled leg swing frequency in this walking support. Besides, Figure 17 shows little overshoot and sufficient convergence at the proportional gain of $k_{* h}=$ 100.0 and the derivative gain of $\hat{k}_{* h}=0.10$ for the unit step response. In consideration of those results, we set the hip joint feedback gains $k_{* h}$ and $\hat{k}_{* h}$ equaled 100.0 and 0.10 in the actual walking supports. In the same way, the sufficient response was observed when the range that $k_{* k}$ was from 100.0 to 200.0 and $\hat{k}_{* k}$ was from 0.10 to 0.20 at less than $1.0 \mathrm{~Hz}$ on the knee joint, 


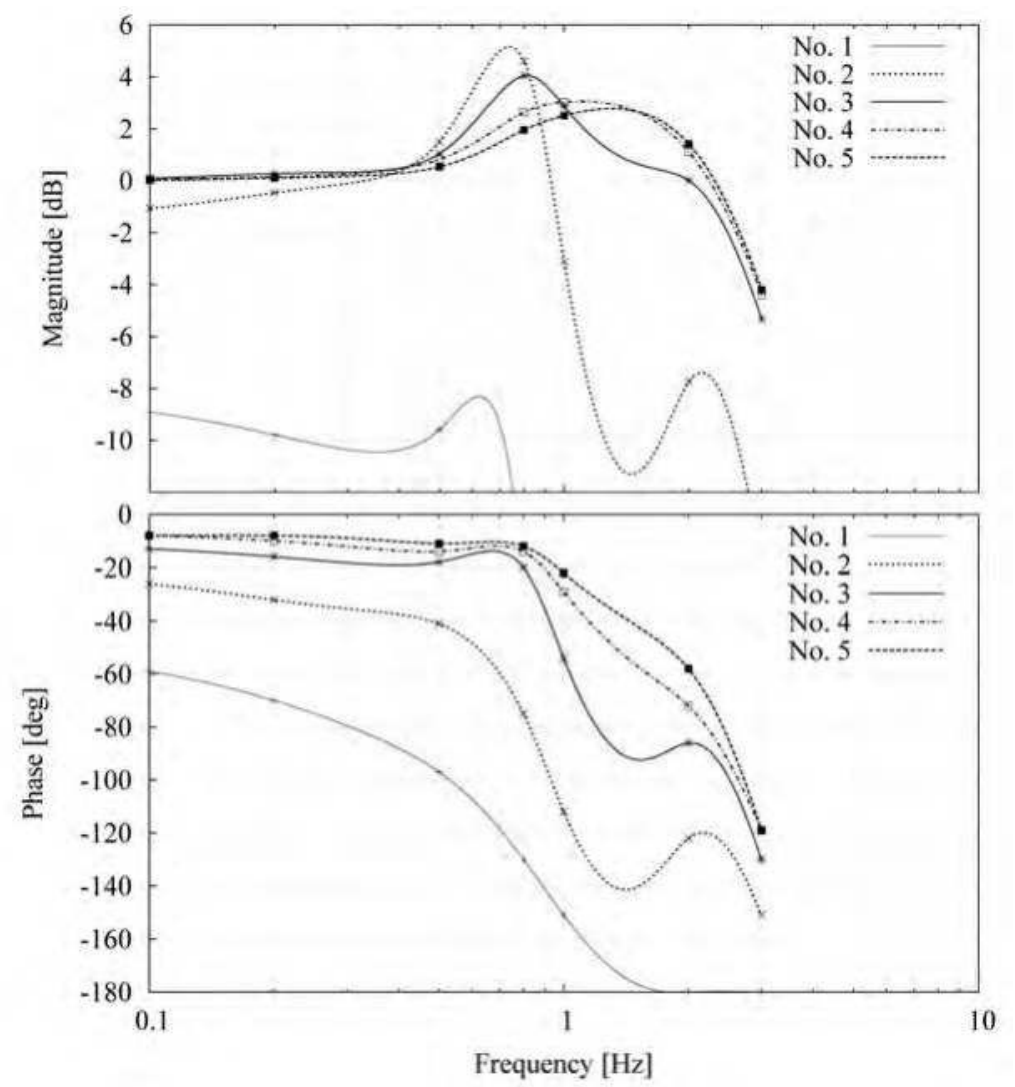

Fig. 16. Frequency response of hip joint shown in Bode plot. Upper graph and lower one show amplitude characteristic and phase characteristic, respectively. Line No. 1 is a characteristic curve in a case of " $k_{* h}=20.0$ and $\hat{k}_{* h}=0.02$ ". Similarly, line No. 2, No. 3, No. 4 and No. 5 show that of " $k_{* h}=50.0$ and $\hat{k}_{* h}=0.05$ ", " $k_{* h}=100.0$ and $\hat{k}_{* h}=0.10 "$ ", " $k_{* h}=150.0$ and $\hat{k}_{* h}=0.15$ " and " $k_{* h}=200.0$ and $\hat{k}_{* h}=0.20$ ", respectively. The all five lines are drawn using a cubic spline curve in order to express the correspondence with seven points in the same set of feedback gains. They therefore interpolate the experimental data and would not precisely express the real values.

as shown in Fig. 18. We also set the knee joint feedback gains of 100.0 and 0.10 from the viewpoint of overshoot and oscillation on the step response, as shown in Fig. 19. 


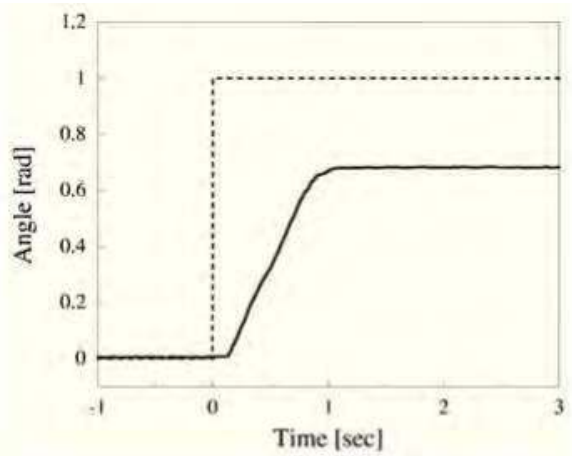

(a) $k_{* h}=20.0, \hat{k}_{* h}=0.02$

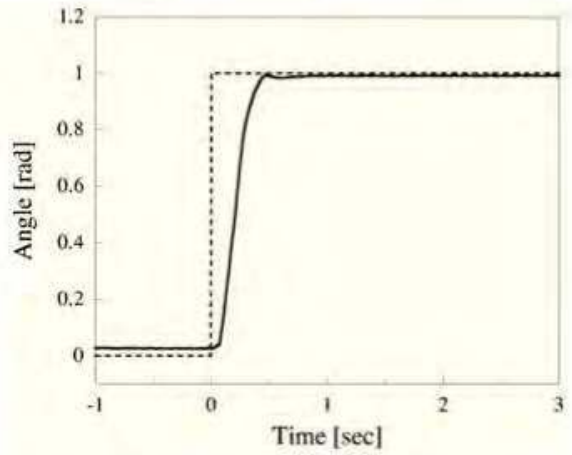

(c) $k_{* h}=100.0, \hat{k}_{* h}=0.10$

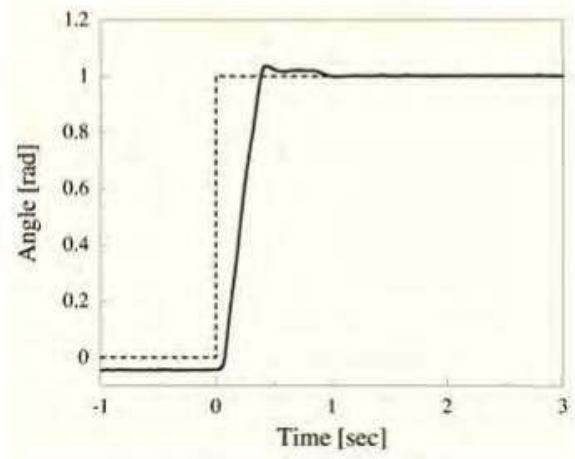

(e) $k_{* h}=200.0, \hat{k}_{* h}=0.20$

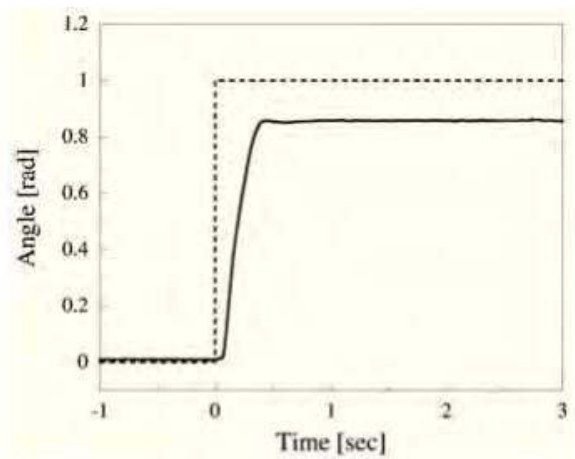

(b) $k_{* h}=50.0, \hat{k}_{* h}=0.05$

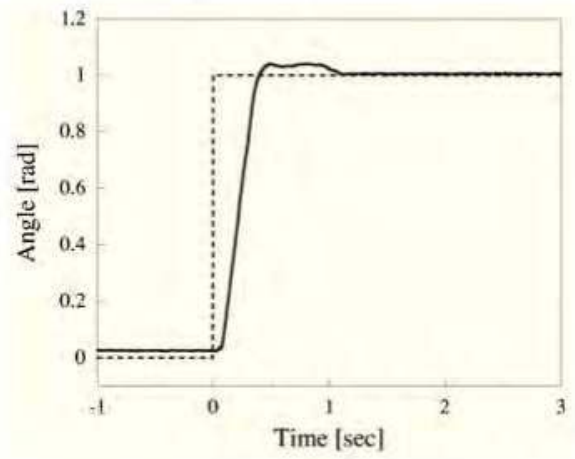

(d) $k_{* h}=150.0, \hat{k}_{* h}=0.15$

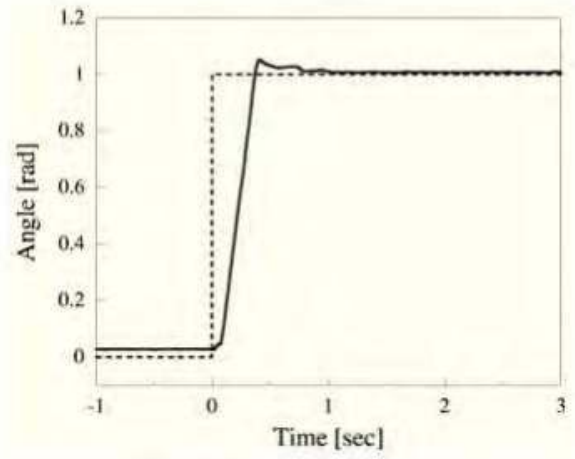

(f) $k_{* h}=300.0, \hat{k}_{* h}=0.30$

Fig. 17. Step responses of hip joint on six kinds of feedback gains (PD gains). Dashed lines and solid lines in the graphs show step inputs and actual angular variations, respectively. 


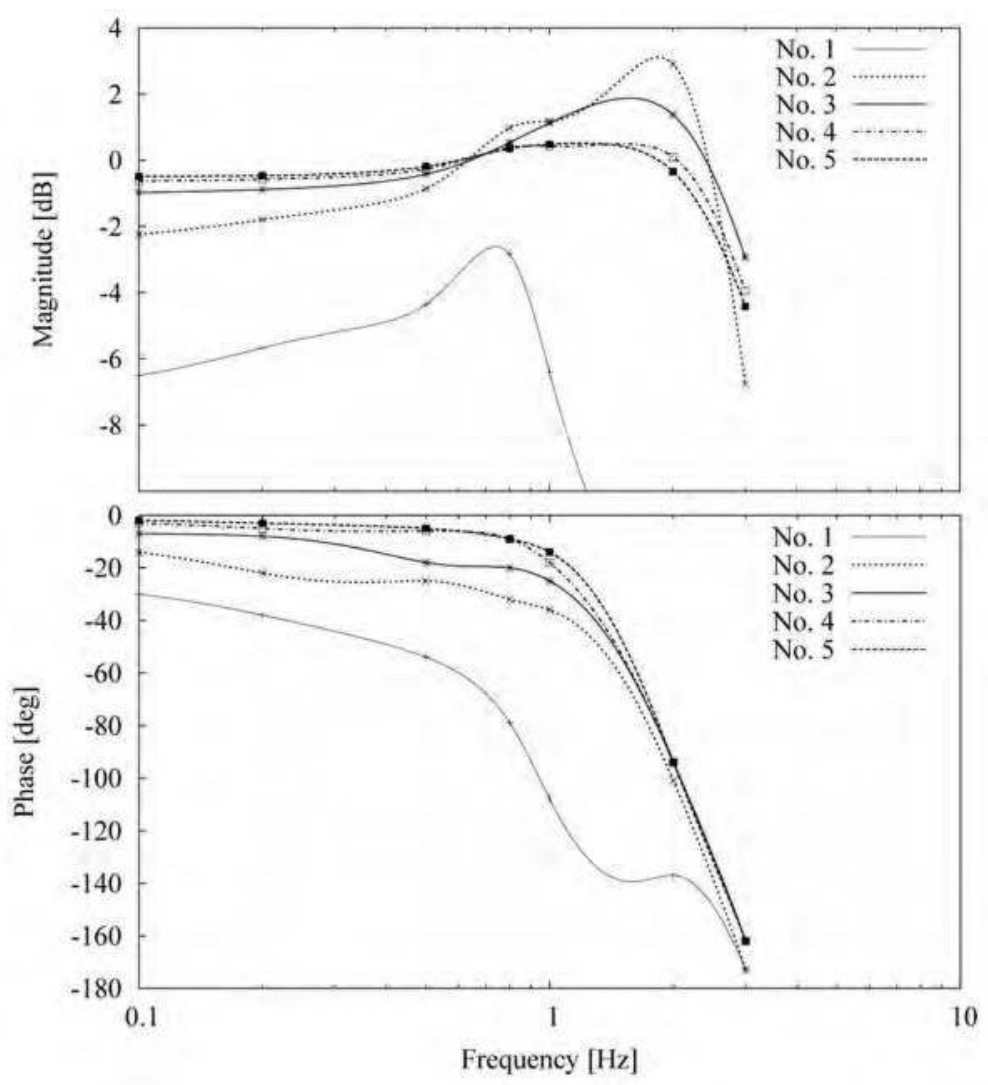

Fig. 18. Frequency response of knee joint shown in Bode plot. Upper graph and lower one show amplitude characteristic and phase characteristic, respectively. Line No. 1 is a characteristic curve in a case of " $k_{* k}=20.0$ and $\hat{k}_{* k}=0.02$ ". Similarly, line No. 2, No. 3, No. 4 and No. 5 show that of " $k_{* k}=50.0$ and $\hat{k}_{* k}=0.05 ", " k_{* k}=100.0$ and $\hat{k}_{* k}=0.10 "$ " " $k_{* k}=150.0$ and $\hat{k}_{* k}=0.15$ " and " $k_{* k}=200.0$ and $\hat{k}_{* k}=0.20 "$, respectively. The all five lines are drawn using a cubic spline curve in order to express the correspondence with seven points in the same set of feedback gains. They therefore interpolate the experimental data and would not precisely express the real values. 


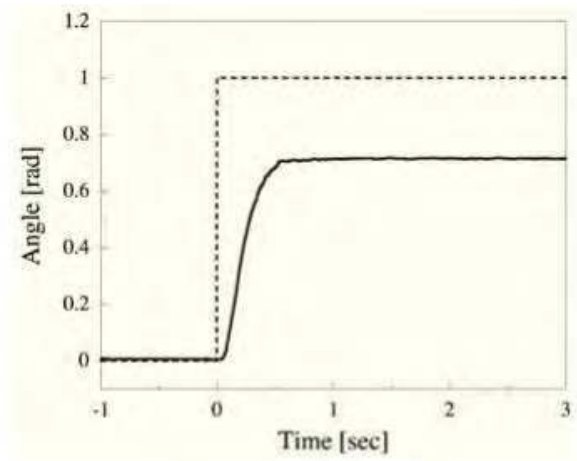

(a) $k_{* k}=20.0, \hat{k}_{* k}=0.02$

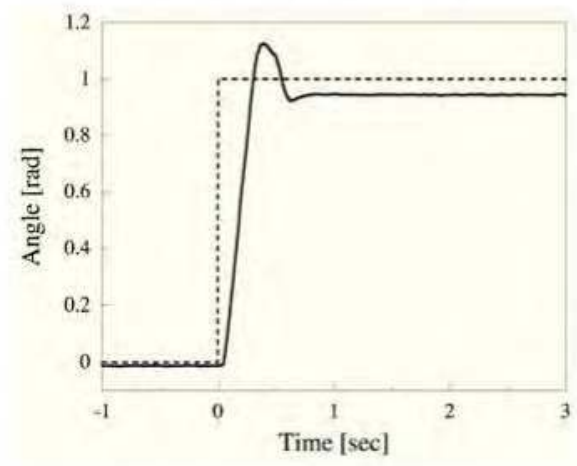

(c) $k_{* k}=100.0, \hat{k}_{* k}=0.10$

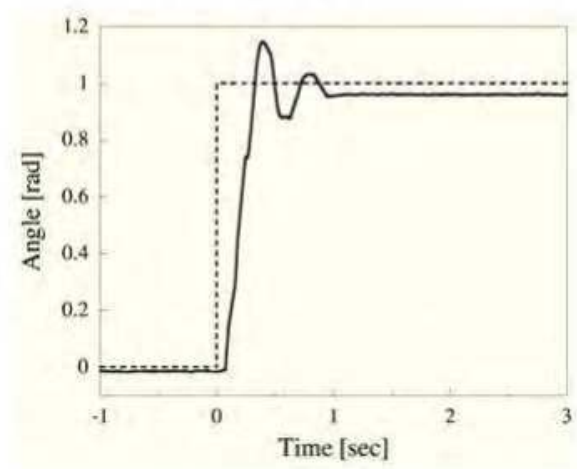

(e) $k_{* k}=200.0, \hat{k}_{* k}=0.20$

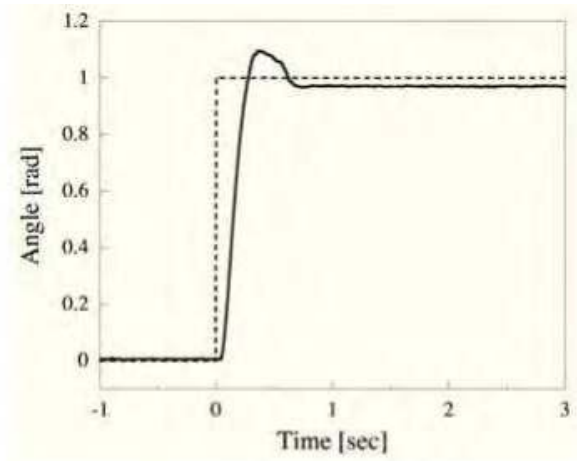

(b) $k_{* k}=50.0, \hat{k}_{* k}=0.05$

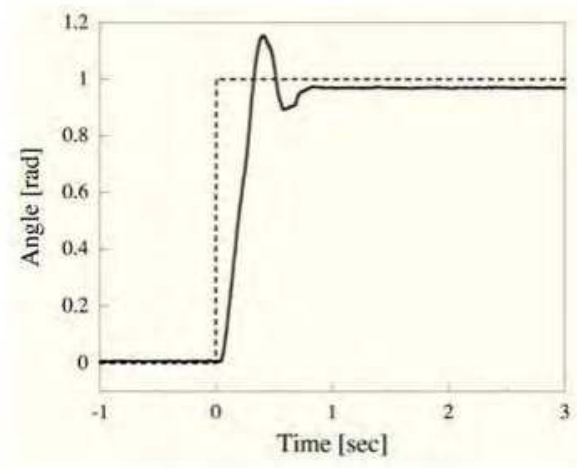

(d) $k_{* k}=150.0, \hat{k}_{* k}=0.15$

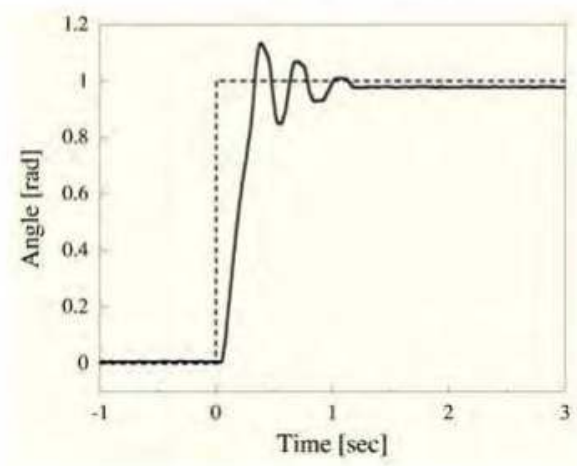

(f) $k_{* k}=300.0, \hat{k}_{* k}=0.30$

Fig. 19. Step responses of knee joints on six kinds of feedback gains (PD gains). Dashed lines and solid lines in the graphs shows step inputs and actual angular variations, respectively. 


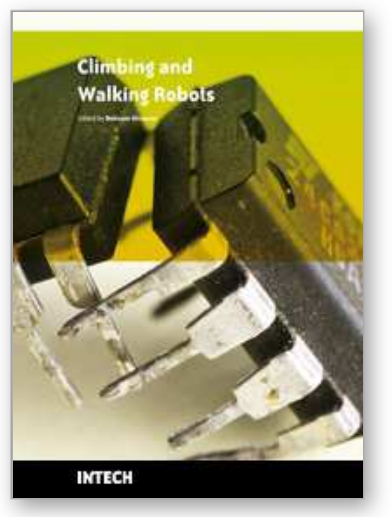

\author{
Climbing and Walking Robots \\ Edited by Behnam Miripour
}

ISBN 978-953-307-030-8

Hard cover, 508 pages

Publisher InTech

Published online 01, March, 2010

Published in print edition March, 2010

Nowadays robotics is one of the most dynamic fields of scientific researches. The shift of robotics researches from manufacturing to services applications is clear. During the last decades interest in studying climbing and walking robots has been increased. This increasing interest has been in many areas that most important ones of them are: mechanics, electronics, medical engineering, cybernetics, controls, and computers. Today's climbing and walking robots are a combination of manipulative, perceptive, communicative, and cognitive abilities and they are capable of performing many tasks in industrial and non- industrial environments.

Surveillance, planetary exploration, emergence rescue operations, reconnaissance, petrochemical applications, construction, entertainment, personal services, intervention in severe environments, transportation, medical and etc are some applications from a very diverse application fields of climbing and walking robots. By great progress in this area of robotics it is anticipated that next generation climbing and walking robots will enhance lives and will change the way the human works, thinks and makes decisions. This book presents the state of the art achievments, recent developments, applications and future challenges of climbing and walking robots. These are presented in 24 chapters by authors throughtot the world The book serves as a reference especially for the researchers who are interested in mobile robots. It also is useful for industrial engineers and graduate students in advanced study.

\title{
How to reference
}

In order to correctly reference this scholarly work, feel free to copy and paste the following:

Kenta Suzuki, Gouji Mito, Hiroaki Kawamoto, Yasuhisa Hasegawa and Yoshiyuki Sankai (2010). IntentionBased Walking Support for Paraplegia Patients with Robot Suit HAL, Climbing and Walking Robots, Behnam Miripour (Ed.), ISBN: 978-953-307-030-8, InTech, Available from: http://www.intechopen.com/books/climbingand-walking-robots/intention-based-walking-support-for-paraplegia-patients-with-robot-suit-hal

\section{INTECH}

open science | open minds

\section{InTech Europe}

University Campus STeP Ri

Slavka Krautzeka 83/A

51000 Rijeka, Croatia

Phone: +385 (51) 770447

Fax: +385 (51) 686166

\section{InTech China}

Unit 405, Office Block, Hotel Equatorial Shanghai

No.65, Yan An Road (West), Shanghai, 200040, China 中国上海市延安西路65号上海国际贵都大饭店办公楼 405 单元

Phone: +86-21-62489820

Fax: +86-21-62489821 
www.intechopen.com 
(C) 2010 The Author(s). Licensee IntechOpen. This chapter is distributed under the terms of the Creative Commons Attribution-NonCommercialShareAlike-3.0 License, which permits use, distribution and reproduction for non-commercial purposes, provided the original is properly cited and derivative works building on this content are distributed under the same license. 